\title{
Radiation therapy after recanalization of esophagus cancer upper thirds
}

\begin{abstract}
Recanalization allows patients to take food by mouth, which is of particular importance during radiation therapy. Watched 35 patients with cancer of the upper third of the esophagus, which after recanalization of the lumen of the authority was held from telegammaterapija that extended, that extended their life from an average of 7.8 months to 15 .
\end{abstract}

Keywords: recanalization, esophagus, radiation, therapy, outcome

\author{
Volume 5 Issue 6 - 2018 \\ Shaposhnikov Veniamin Ivanovich \\ Department of Oncology, Russia
}

Correspondence: Shaposhnikov VI, Department of Oncology with a course of radiation Diagnostics and therapy the NIGHT, Kuban Medical Institute, Krasnodar, Russia, Email 79183446404@yandex.ru

Received: June 15, 2018 | Published: November 26, 2018

\section{Materials and methods}

Include a group of patients with unresectable esophageal cancer, which was held on telegammaterapija standard method after previously executed recanalization of the lumen of the body. Such treatment was carried out 35 patients with unresectable cancer medium-the upper third of the esophagus. A plastic tube was implemented long recanalization $30-40 \mathrm{~cm}$, diameter $16 \mathrm{~mm}$, a wall thickness of $0.7 \mathrm{~mm}$. At one end it had a diameter of $25 \mathrm{~mm}$ extension is funnel-shaped, covered outdoor taping of foam which fit in case soldered down plastic sheeting. Operation performed under jendotrahealnym anesthesia with controlled breathing. Extension of the esophagus produce buzhami increasing calibers. For this gastrotomiju and then served from the lumen of the stomach, in a direction from below upwards, the lumen of the esophagus were introduced. As soon as the end of

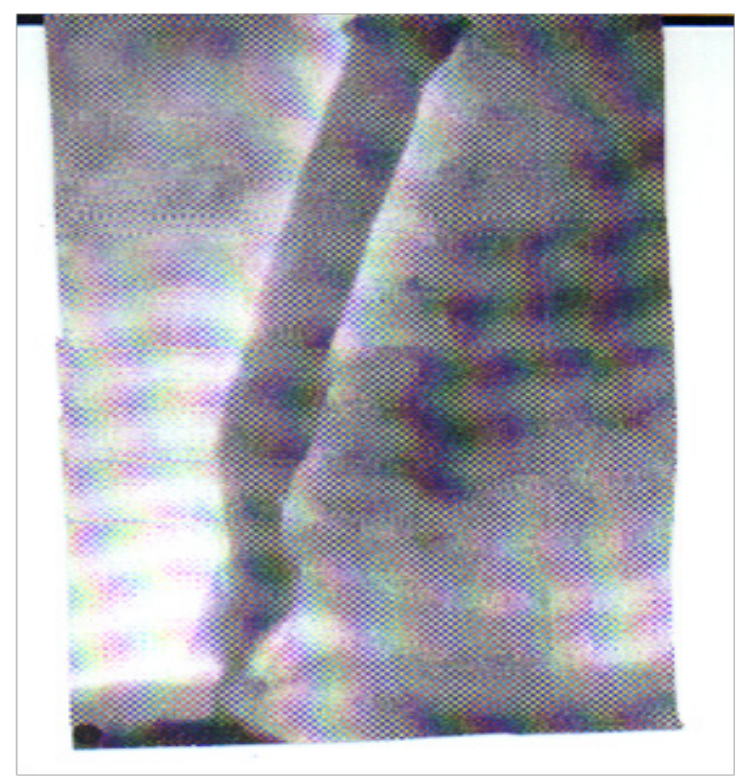

Figure I Radiographs of the esophagus after his recanalization. the burza (storm) appeared in oropharyngeal polsti, he was drawn out. Following this, he tied a long lavsanovye. And then they reverse traction burza (storm), conducted in the lumen of the stomach. To the ends of these strands, protruding from the mouth, fixed prosthesis. Careful sipping for the ends of the thread held in your stomach and with the participation of an anesthesiologist, a tube was introduced through the throat into the esophagus until it stops extended her part in the upper pole of the tumor. Overweight the bottom end otsekalsja prosthesis in the cavity of the stomach. To create flexibility, while maintaining the rigidity, projecting from the esophagus to the stomach part tube caused 4-5 side slots. Zashivalas in the stomach wound tightly. Accepting liquid food the patient started the day after surgery. And after 2-3 day started by the Pasty, squeezed her water. To beam treatment started on the 2-3 day after surgery and performed it in full (Figure $1 \&$ Figure 2).

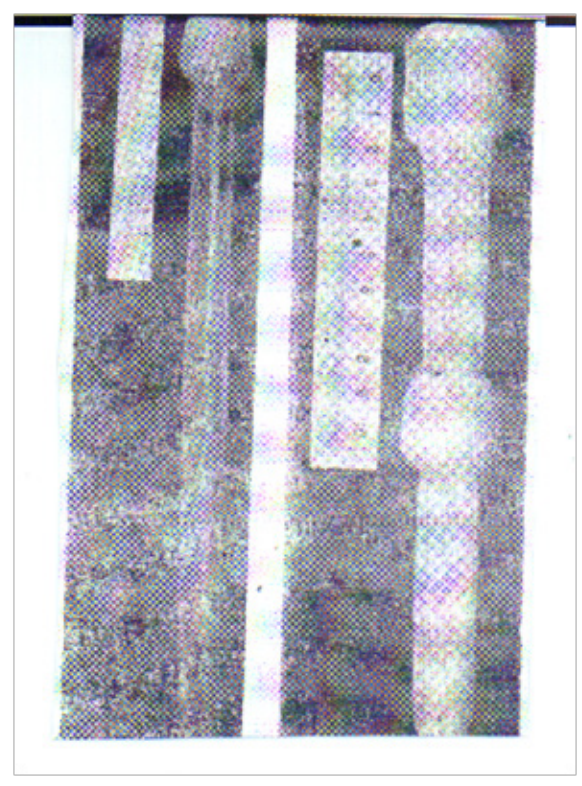

Figure 2 Tube recanalization. 


\section{Results and discussion}

The effectiveness of the method was evaluated by postoperative average life expectancy. It is equal to 15 months, while 44 have patients with gastrostomiej, only 7, 8 months. The technique not gastrostomiej influenced this statistic. Among the specific complications can include moderate bleeding when the paper passes the tube through the lumen of the tumor, which quickly stopped independently, as well as clogging lumen tube dense food. This happened on 2 patients through 2-3 months after the operation. Tubes pass ability re-established by her reconstruction. ${ }^{1-7}$

\section{Conclusion}

Esophageal Rekanalizaciju you can assess how effective palliative surgical intervention that saves patients from dysphagia until the last minutes of their lives, and thereby enables them to radiation treatment. If there is no Skin maceration and other pathological processes inherent in the gastrostomiej.

\section{Acknowledgments}

None.

\section{Conflicts of interest}

The author declares that there is no conflicts of interest.

\section{References}

1. Borodin IM. Recanalization of the esophagus and cardia in inoperable cancer. Herald of Surgery.1963;90(1):55-57.

2. Starikov VI. Cancer of the esophagus: diagnosis and treatment prospects. International Medical Journal. 2006;12(1):66-70.

3. Chernousov AF. Surgery of the esophagus. Medicine. 2000:350.

4. Shevchenko IT, Shaposhnikov VI. Cancer of the esophagus. Medicine. 1972:124.

5. Berrisford RG. Short-term otitcomes following total minimally invasive oesophagectomy. Br J Surg. 2008;95(5):602-610.

6. Das A. A Comparison of Endoscopic Treatment and Surgery in Early Esophageal Cancer. An Analysis of Surveillance Epidemiology and End Results Data. Am J Gastroenterol. 2008;103(6):1340-1345.

7. Coyas A. Palliative intubation in carcinoma of otsophagus. Lancet. 1955;2(13):81-88. 